\title{
The Fraction of Ionizing Photons Escaping from High-Redshift Galaxies
}

\author{
Jhan A. Srbinovsky ${ }^{\mathrm{A}}$ and J. Stuart B. Wyithe $\mathrm{A}^{\mathrm{A}, \mathrm{B}}$ \\ A School of Physics, University of Melbourne, Parkville, Victoria, Australia \\ ${ }^{B}$ Corresponding author. Email: swyithe@ unimelb.edu.au
}

Received 2009 June 28, accepted 2009 December 10

\begin{abstract}
The fraction $\left(f_{\text {esc }}\right)$ of ionizing photons that escape their host galaxy and so are able to ionize hydrogen in the intergalactic medium is a critical parameter in studies of the reionization era. In this paper we constrain $f_{\text {esc }}$ at high redshift by modelling the observed Ly $\alpha$ transmission. Using an $N$-body simulation of the cosmic web, we first model an ionizing background due to star-bursting galaxies. We then extract an ensemble of mock absorption spectra from the simulation and compute the mean transmission. We adjust the free parameter, $f_{\text {esc }}$ in our model of the ionizing background to reproduce the observed transmission. We find that $f_{\text {esc }}$ increases with the minimum galaxy mass considered to contribute to the ionizing background, and that at high redshift $f_{\text {esc }}$ must be greater than 5\% irrespective of galaxy mass. Based on our numerical results we calibrate a semi-analytic model which shows that the ionizing background observed at $z \sim 5.5-6$ implies a sufficient number of ionizing photons to have reionized the Universe by $z \sim 6$. However, if the minimum mass for star-formation were $\gtrsim 10^{9} \mathrm{M}_{\odot}$, the ionizing background would be over-produced at $z \lesssim 5$. In summary, our results indicate that the Universe was reionized by low mass galaxies.
\end{abstract}

Keywords: cosmology: diffuse radiation — galaxies: high redshift — intergalactic medium — large scale structure of Universe

\section{Introduction}

The sources thought to have produced the UV radiation, which reionized the hydrogen gas in the inter-galactic medium (IGM), are star-bursting galaxies and quasars (e.g. Barkana \& Loeb 2001). However, the number density of the quasar population is observed to decline exponentially with redshift at $z \gtrsim 2.5$, implying that galaxies probably contribute the bulk of UV photons that drive both reionization at $z \gtrsim 6$ and also evolution of the ionizing background, post-overlap, at $z \sim 3-6$ (Madau, Haardt \& Rees 1999; Fan et al. 2002; Bolton \& Haehnelt 2007; Srbinovsky \& Wyithe 2007). The potential contribution of galaxies to the UV radiation field is a function of their number density, the star formation rate (SFR) within the galaxies and the spectral energy distribution (SED) of the stellar population. However, the fraction of ionizing photons which escape their host galaxy into the IGM, is limited by intervening absorption in the interstellar medium (ISM). The value of this escape fraction $\left(f_{\text {esc }}\right)$ is therefore a critical parameter in studies of reionization, and remains relatively uncertain.

Owing to its importance, constraints on the value of the escape fraction feature extensively in the literature. However, the definition of $f_{\text {esc }}$ varies depending on the study. A tendency exists in observational studies to quote values of $f_{\text {esc }}$ as the escape fraction of Lyman-limit photons, relative to the escape fraction at $1500 \AA\left(f_{\mathrm{esc}}^{1500}\right)$. In this paper, we define $f_{\text {esc }}$ as the absolute escape fraction of photons at the Lyman limit, since this is the parameter of physical importance for the reionization history of hydrogen. Where necessary, we convert values of the escape fraction from published studies to be consistent with this definition, assuming $f_{\mathrm{esc}}^{1500}=0.24$ and an extinction with a median value of $E(B-V)=0.15$ at $z \lesssim 3$ (Siana et al. 2007).

Early theoretical studies predicted values for the escape fraction of between $f_{\mathrm{esc}} \sim 3$ and $15 \%$ (Dove \& Shull 1994; Dove, Shull \& Ferrara 2000; Ciardi et al. 2002; Fujita et al. 2003), however Clarke \& Oey (2002) suggested that $f_{\text {esc }}$ could be much higher than this if the feedback dependent porosity of the interstellar medium is considered. At high redshift, Fujita et al. (2003) argue that increased supernovae activity create escape tunnels through the interstellar medium for ionizing radiation, and estimate $f_{\text {esc }}>20 \%$ by $z>5$. Similarly, Razoumov $\&$ Sommer-Larsen (2006) find that $f_{\text {esc }}$ evolves from $\sim 1-$ $2 \%$ at $z=2.39$ to $\sim 6-10 \%$ at $z=3.6$. In contrast, Wood \& Loeb (2000) suggest that $f_{\text {esc }}$ decreases with redshift (becoming $\sim 1 \%$ at $z \sim 10$ ) due to the increasing density of the galactic disk. At $z \sim 20$, Whalen, Abel \& Norman (2004) and Alvarez et al. (2006), have derived escape fractions as high as $f_{\text {esc }} \sim 95 \%$, however in these cases the large value can be attributed to the assumed Population-III stellar population. Finally, Kitayama et al. (2004) studied the evolution of HII regions surrounding a central Population-III star embedded in a galaxy and find that $f_{\text {esc }}$ increases with the mass of the star and inversely with the mass of the halo. 
Observationally, the escape fraction of ionizing photons from the Milky Way galaxy can be reliably measured by modeling the observed emission measures and kinematics of the Magellanic stream in the presence of photoionization due to hot, young stars in the Galactic disk, yielding a value of $\sim 6 \%$ (Bland-Hawthorn \& Maloney 1999). In the local Universe, observational estimates of $f_{\text {esc }} \sim 2-11 \%$, have been derived ${ }^{1}$ from measurements of emission line strengths relative to the Lyman-limit flux (Hurwitz, Jelinsky \& Dixon 1997; Heckman et al. 2001; Bergvall et al. 2006; Grimes et al. 2007). An alternative approach measures the flux in the Lyman-continuum relative to the flux at a more readily detectable frequency above the Lyman limit (usually $1500 \AA$ ). Comparison is then made to the intrinsic flux ratio predicted by an assumed SED of galaxies, which can be characterized by the flux decrement $(L B)$ across the Lyman break. Assuming $L B=3$, Deharveng et al. (2001) estimate values of $f_{\text {esc }} \lesssim 10 \%$ in nearby galaxies, and Malkan, Webb $\&$ Konopacky (2003) estimate values of $f_{\text {esc }} \lesssim 1-6 \%$ from a sample of 11 blue galaxies at $z \sim 1$. At $z \sim 3$, Steidel et al. (2001) compile a composite spectrum from 29 Lyman-break galaxies and find an average escape fraction of $f_{\text {esc }}>10 \%$, and from observations of 2 galaxies Giallongo et al. (2002) determine values of $f_{\text {esc }} \lesssim 4 \%$. From a sample of 14 star-forming galaxies, Shapley et al. (2006) determines the escape fraction to be $f_{\mathrm{esc}} \sim 3 \%$, however, in two of these galaxies detection of Lyman continuum flux suggests an escape fraction of the order of $10 \%$. A less stringent constraint is found by Inoue et al. (2005), who find the escape fraction to be $f_{\text {esc }} \lesssim 20-40 \%$ at $z \sim 3$ using narrow band images of two galaxies. Finally, the escape fraction has also been constrained by Schaye (2006) who found $f_{\mathrm{esc}} \sim 10 \%$ at $z \sim 3$, by comparing the absorption systems near an ionizing source with those in the average IGM.

Alternatively, if an SED is available for the galaxy, then the the effects of intrinsic and intervening absorption can be modelled. Using this method Fernández-Soto, Lanzetta \& Chen (2003) find $f_{\text {esc }} \leq 4 \%$, by fitting to the observed SEDs of 27 galaxies at $1.9 \leq z \leq 3.5$. Similarly, at $z \sim 1$, Siana et al. (2007) fit optical/near-infrared model SEDs to the observed spectra of 21 galaxies and finds the average, intrinsic Lyman-break factor to be $\sim 6$, from which they conclude the global escape fraction to be, $f_{\text {esc }} \lesssim 4 \%$. Recently, Chen et al. (2007) estimate $f_{\text {esc }} \sim 2 \%$ at $z \sim 2$ from the observed distribution of neutral hydrogen column densities in the after-glow spectra of long duration GRBs. This approach alleviates the dependence on largely unknown intrinsic spectral properties of the galaxy, however presents another issue of a possible GRB environment bias. Finally, combining estimates of $f_{\text {esc }}$ from published studies with values derived from measurements of the ionizing background, Inoue, Iwata \& Deharveng (2006) find

\footnotetext{
${ }^{1}$ In this range for $f_{\text {esc }}$ we have excluded the finding of Hurwitz et al. (1997) for one of the four galaxies in their studied sample in which $f_{\text {esc }} \lesssim 57 \%$.
}

that the escape fraction increases from $f_{\mathrm{esc}} \sim 1$ to $10 \%$ in the redshift interval $1 \lesssim z \lesssim 4$.

While there is as yet no consensus on the value of $f_{\text {esc }}$, the implications for the reionization history have been addressed by several authors. Recently, detailed numerical simulations Gnedin, Kravtsov \& Chen (2008) have predicted a value for $f_{\mathrm{esc}}$ of between 1 and $3 \%$, from halos of mass $M \gtrsim 5 \times 10^{10} \mathrm{M}_{\odot}$ within the redshift range $3<z<9$. In addition to a small escape fraction in massive galaxies Gnedin et al. (2008) further predict that halos with $M \lesssim 5 \times 10^{10} \mathrm{M}_{\odot}$ have an escape fraction which is negligibly small. This very low efficiency of reionization would have profound effects on the reionization history. Indeed, Gnedin (2008) argues that the small escape fraction for lower mass galaxies implies that the observed galaxy population at $z \sim 6$ could not have produced enough UV photons to reionize the IGM. This suggests that the process of reionization takes place in a 'photon starved' environment. This conclusion has been previously reached by Bolton \& Haehnelt (2007) who studied the ionizing background based on the Ly $\alpha$ absorption properties towards high redshift quasars. However, Bolton \& Haehnelt (2007) inferred a higher a value for the escape fraction of $f_{\text {esc }} \sim 20-30 \%$ (at $z \sim 6$ ).

Recently, estimates of $f_{\text {esc }} \sim 0.5 \%$ (at $z \sim 2-4$ ) have been reported by Faucher-Giguere et al. (2008). This low estimate however reflects a very hard SED, extrapolation of the LF to zero luminosity and the assumption that above the Lyman limit the intrinsic number of photons produced by the galaxies is equal to the number of photons which are observed.

In this paper we employ an approach that is similar to Bolton \& Haehnelt (2007), and constrain the escape fraction of ionizing photons (at $z \sim 5.5-6$ ) by combining the observed LF of galaxies with the observed opacity of the high redshift IGM. However, with respect to the study of the escape fraction our modelling includes several improvements. We employ an $N$-body simulation to describe the density and peculiar velocity fields and to identify virialized halos. We determine the UV luminosity of each halo using a semi-analytic description and constrain model parameters by fitting to the observed galaxy LF. We then generate an inhomogeneous ionizing background using these discrete galaxies, which depends on the escape fraction of ionizing radiation. For a range of escape fractions we extract an ensemble of mock Ly $\alpha$ absorption spectra along 3072 sight-lines through our simulation box and determine the value of $f_{\text {esc }}$ which best models the observed Ly $\alpha$ transmission. We note that in our model the escape fraction is not explicitly dependent on galaxy mass or angular direction.

The paper is organized as follows. In Section 2 we outline our models for the ionizing background due to discrete galaxies. We also describe our computation of the Lya photon transmission. In Section 3 we show our results for $f_{\text {esc }}$ at several redshifts assuming mean-free-paths (MFP) which are consistent with the observationally derived values from Fan et al. (2006). In Section 4 we repeat 
our analysis to constrain $f_{\text {esc }}$ assuming the suppression of galaxy formation in low mass halos $\left(M<10^{10} \mathrm{M}_{\odot}\right)$, while in Section 5 we exclude halos with $M<5 \times 10^{10} \mathrm{M}_{\odot}$. In Section 6 we extrapolate our numerical results to other minimum galaxy masses. In Section 7 we discuss the implications of our results using a semi-analytic model of reionization history. Finally we make some concluding remarks in Section 8. Throughout the paper we assume a $\Lambda \mathrm{CDM}$ cosmology with parameters $\left(\sigma_{8}, h, \omega_{\mathrm{B}}\right.$, $\left.\omega_{\mathrm{M}}, \omega_{\Lambda}\right)=(0.8,0.7,0.04,0.3,0.7)$. All distances are in co-moving units unless otherwise stated.

\section{Modelling Ly $\alpha$ Transmission and a Fluctuating Ionizing Background}

A brief summary of our approach to constrain the escape fraction is outlined below, followed by several subsections describing our model in more detail. We employ an $N$-body simulation to compute the density and peculiar velocity fields and also to locate dark matter (DM) halos, which we then associate with galaxies. To a fraction of these galaxies which are considered to be actively starbursting, we assign a luminosity (at $\lambda=1350 \AA$ ) based on models for the star formation rate (SFR) and the intrinsic SED. We constrain parameters in these models by measuring the LF from the simulation and fitting to the observed LF. Assuming the ionizing background to be dominated by galaxies (see Section 1), we next compute the luminosity of these actively UV luminous galaxies at the Lyman limit, and using a simple model generate a field of ionizing radiation which reflects the distribution of these discrete sources. We are then able to compute an ensemble of Ly $\alpha$ Forest spectra along lines-of-sight through our simulation volume, from which we can measure the mean transmission of the IGM. Our model for describing the ionizing background contains the parameter $f_{\text {esc }}$, which we adjust so that the mean transmission measured from our model matches the observed mean transmission.

\subsection{The N-Body Simulation}

The simulation followed $1024^{3}$ DM particles in a cubic volume of side-length, $L=65.6 \mathrm{Mpc} / h$. Snap-shots of the density and peculiar velocity fields were taken periodically in redshift space with bound halos identified using a friends-of-friends algorithm. The simulation accurately resolves halos with mass, $M>2 \times 10^{9} \mathrm{M}_{\odot}$. We place the DM halos and the density and velocity fields onto a $512^{3}$ grid. In this paper we consider redshift snap-shots of the density and peculiar velocity fields at redshifts $z=5.5,5.7,6.0$. The numerical simulation assumed $\sigma_{8}=0.9$ which we translate to a lower value of $\sigma_{8}$ following McQuinn et al. (2007). Further details of the numerical simulation can be found in Zahn et al. (2007); and Lidz et al. (2007).

Baryonic gas pressure is not included in our (DM only) $N$-body simulation. However Hydro-Particle-Mesh (HPM) simulations can be used to approximate the effect of gas pressure. Gnedin \& Hui (1998) show that HPM simulations of the IGM are capable of reproducing measurable quantities (such as absorption spectra) with a precision comparable to full hydro-dynamic simulations. Though less reliable than HPM simulations, Gnedin \& Hui (1998) also show that the effect of pressure can be incorporated in $N$-body simulations by smoothing the linear density field on a suitable filtering scale $\left(k_{\mathrm{f}}\right)$. Lidz, Oh \& Furlanetto (2006) measured the power spectrum of Ly $\alpha$ transmission fluctuations $P_{\mathrm{F}}(k)$, using HPM simulations to describe the density and velocity fields. To determine the correct filtering scale for our $N$-body simulation we therefore measure $P_{\mathrm{F}}(k)$ following smoothing of the density field on a range of filtering scales. We find the best agreement with Lidz et al. (2006) is obtained for a filtering scale of $k_{\mathrm{f}}=20 \mathrm{Mpc}^{-1}$, and adopt this value throughout the paper.

\subsection{The Ionizing Background}

The fraction of galaxies identified in our simulation which are actively star-bursting at a particular redshift, can be described by the duty-cycle of the star-burst $\left(t_{\mathrm{DC}}=t_{\mathrm{S}} / t_{\mathrm{H}}\right.$, where $t_{\mathrm{H}}$ is the Hubble time at $z$ ). To this fraction of galaxies we assign a luminosity (at $\lambda=1350 \AA$ ) by first estimating the SFR which (following Loeb, Barkana \& Hernquist 2005) is dependent on the mass of the halo $(M)$, the star-formation efficiency $\left(f^{\star}\right)$ and again the duration of the star-burst $\left(t_{\mathrm{s}}\right)$.

$$
\begin{aligned}
\mathrm{SFR}= & 0.17\left(\frac{M}{10^{9} \mathrm{M}_{\odot}}\right)\left(\frac{t_{\mathrm{S}}}{10^{8} \mathrm{yr}}\right)^{-1} \\
& \times\left(\frac{f^{*}}{0.1}\right)\left(\frac{\Omega_{\mathrm{B}} / \Omega_{\mathrm{M}}}{0.17}\right) \mathrm{M}_{\odot} \mathrm{yr}^{-1} .
\end{aligned}
$$

The specific luminosity at wavelength $\lambda$ of each halo can then be computed from

$$
L_{\nu}(\lambda)=j_{\mathrm{L}}(\lambda) \frac{\mathrm{SFR}}{1 \mathrm{M}_{\odot} \mathrm{yr}^{-1}} \operatorname{erg~s}^{-1} \mathrm{~Hz}^{-1},
$$

where $j_{\mathrm{L}}(\lambda)$ is the specific rest frame luminosity, per $\mathrm{Hz}$, per SFR (solar masses per year). We compute $j_{\mathrm{L}}(\lambda)$ using a model for SED of star-bursting galaxies which is presented in Leitherer et al. (1999). We assume metal enriched stars of 0.05 solar metallicity, a Scalo (1998) IMF (for 1-100 $\mathrm{M}_{\odot}$ ) and continuous star-formation over a star-burst lifetime of $100 \mathrm{Myr}$. This low solar metallicity is reasonable at high redshift and this star-burst lifetime is consistent with the duty-cycle which is constrained below. The relative flux density between 1500 and $900 \AA$ for this SED is given by $L B \sim 3$.

As our aim is to reproduce the observed LF we correct this intrinsic source luminosity by including extinction by dust within the galaxy. We adopt a factor of 1.5 for the ratio of the intrinsic to the observed luminosity (at $z \sim 6$ ) following Bouwens et al. (2007, and references therein). For 10000 combinations of $t_{\mathrm{s}}$ and $f^{\star}$ we compute the $\mathrm{LF}$ in units of the number of galaxies per AB-magnitude per co-moving volume $\left(\mathrm{d} n / \mathrm{d} M_{1350}\right)$ and constrain these parameters using a maximum likelihood technique, fitting our model LF to the observed LF of Bouwens et al. (2007) 


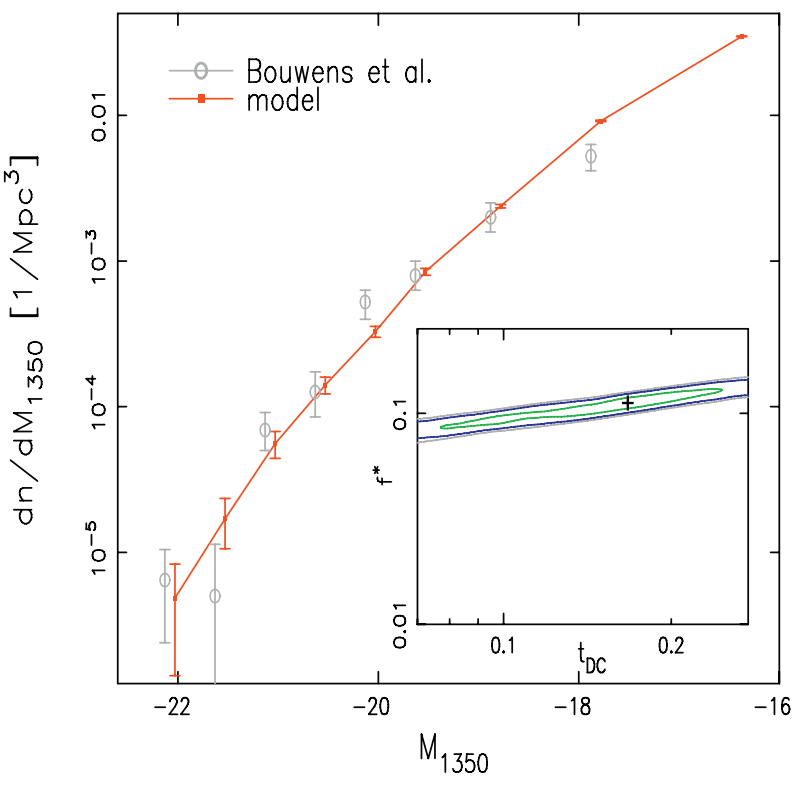

Figure 1 The best fit model $\mathrm{LF}$ with parameter values of $t_{\mathrm{DC}}=0.16$ $\left(t_{\mathrm{S}}=2.2 \times 10^{8} \mathrm{yr}\right)$ and $f^{\star}=0.11$, as constrained by a maximum likelihood analysis. Inset is a plot of the likelihood distribution in which the contours represent the $1,2,3-\sigma$ confidence levels with a cross to mark the point of maximum likelihood. For display purposes a line joins the model points (solid). The error bars on the model reflect the number of galaxies in each luminosity bin as measured from our simulation. The open circles represent the data from the observed UV galaxy LF (Bouwens et al. 2007). The model points are shifted slightly to the right of the observational data for clarity only.

at $z=6$. We assume flat prior probability distributions for the parameters $t_{\mathrm{DC}}$ and $f^{\star}$. In Figure 1 we show the best fit LF. For display purposes a line joins the model points (solid) which are the centers of the constructed luminosity bins. At the faint end of the LF we have constructed an additional bin as within this star-formation model, the simulation resolves galaxies down to a lower luminosity than are currently observed. The error bars on the model reflect the number of galaxies in each luminosity bin. The open circles represent the data from the observed UV galaxy LF (Bouwens et al. 2007). The model points are shifted slightly to the right of the observational data for clarity. Inset in Figure 1 is a plot of contours representing the 1- $\sigma$, 2- $\sigma$ and 3- $\sigma$ confidence levels on the parameters $f^{\star}$ and $t_{\mathrm{DC}}$. A cross marks the point of maximum likelihood, with values of $t_{\mathrm{DC}}=0.16\left(t_{\mathrm{S}}=2.2 \times 10^{8} \mathrm{yr}\right)$ and $f^{\star}=0.11$.

In this model the minimum mass halo $(M \sim 2 \times$ $10^{9} \mathrm{M}_{\odot}$ ) which contributes to the ionizing background corresponds to a specific luminosity of $L \sim 8.9 \times$ $10^{26} \mathrm{erg} \mathrm{s}^{-1} \mathrm{~Hz}^{-1}$ (at $1350 \AA$ ) and a magnitude of $M_{1350} \sim-15.8$. The largest halo identified in our simulation has a mass of $M \sim 3 \times 10^{12} \mathrm{M}_{\odot}$, which given the constrained parameters corresponds to a specific luminosity of $L \sim 1.2 \times 10^{30} \mathrm{erg} \mathrm{s}^{-1} \mathrm{~Hz}^{-1}$ and a magnitude of $M_{1350} \sim-23.8$. However at $z=6$, only $\sim 13 \%$ of identified halos have masses greater than $1 \times 10^{10} \mathrm{M}_{\odot}$ and only $\sim 1 \%$ have masses greater than $5 \times 10^{10} \mathrm{M}_{\odot}$. The largest actively star-bursting halo selected in our simulation has a mass of $M \sim 1 \times 10^{12} \mathrm{M}_{\odot}$, which corresponds to a specific luminosity of $L \sim 4.4 \times 10^{29} \mathrm{erg} \mathrm{s}^{-1} \mathrm{~Hz}^{-1}$ and a magnitude of $M_{1350} \sim-22.5$. We note that in our model the minimum luminosity halo that contributes to the UV background is equivalent to the definition of the minimum luminosity cut off in the integration of the LF which is used to derive the global emissivity due to galaxies in other studies (e.g. Bolton \& Haehnelt 2007).

Using the constrained values of $f^{\star}$ and $t_{\mathrm{DC}}$, we reevaluate the luminosity of each star-bursting galaxy at the Lyman limit (912 $\AA$ ). We then re-position the UV luminous halos at the center of the simulation cell in which they reside and construct a luminosity grid $L(x)$ as a function of position, which matches the dimensions of our density and velocity grids from the simulations. As the grid is relatively fine $(65.6 / 512 \mathrm{Mpc} / h)$ the effect of this re-positioning on the resulting ionizing background is negligible in comparison to other uncertainties in our model (such as the assumed MFP). More significantly however, the sources remain correlated with the over-density field, which is ultimately the desired effect here. Very occasionally more than one halo resides within the boundaries of a cell. In this case we consider a single more massive halo at the center of the cell.

Given the positions and luminosities of the galaxies we are able to compute the flux at every position on our grid using a procedure which is similar to that in Bolton et al. (2005); Bolton \& Haehnelt (2007); McDonald et al. (2005). We first compute the co-moving energy density $(\mathrm{d} E / \mathrm{d} V)$ at the Lyman limit through the Fourier convolution

$$
\frac{\mathrm{d} E}{\mathrm{~d} V}\left(\boldsymbol{x}^{\prime}\right)=\frac{1}{V_{\text {cell }}} \int_{-\infty}^{\infty} \mathrm{d} \boldsymbol{x} L(\boldsymbol{x}) G\left(\boldsymbol{x}^{\prime}-\boldsymbol{x}\right),
$$

which conveniently accounts for flux contributions from galaxies across the periodic boundaries in our simulation box. In this equation $V_{\text {cell }}$ is the co-moving volume of a grid cell and the attenuation of photons as they propagate from sources is described by the filter function $G\left(\boldsymbol{x}^{\prime}-\boldsymbol{x}\right)$, which is defined as

$$
G\left(\boldsymbol{x}^{\prime}-\boldsymbol{x}\right)=\frac{e^{-\left|\boldsymbol{x}^{\prime}-\boldsymbol{x}\right| / \lambda_{\mathrm{mfp}}}}{4 \pi\left(\boldsymbol{x}^{\prime}-\boldsymbol{x}\right)^{2} c(1+z)},
$$

where $\lambda_{\text {mfp }}$ is the MFP of ionizing photons, $\boldsymbol{x}^{\prime}$ is the position at which the energy density is to be evaluated, $\boldsymbol{x}$ is the position of the source, and $c$ is the speed of light. We then compute the flux in physical units of $J_{21}\left(\mathrm{erg} \mathrm{Hz}^{-1} \mathrm{~s}^{-1} \mathrm{~cm}^{-2} \mathrm{sr}^{-1}\right.$ ) from the co-moving energy density.

$$
J_{21}\left(\boldsymbol{x}^{\prime}\right)=\frac{f_{\mathrm{esc}}(1+z)^{3}}{10^{-21}} \frac{c}{4 \pi} \frac{\mathrm{d} E}{\mathrm{~d} V}\left(\boldsymbol{x}^{\prime}\right) .
$$

Here, the (free) parameter $f_{\text {esc }}$ represents the fraction of ionizing photons produced during star-formation which escape the host galaxy. An important simplification in the 
construction of our ionizing background is the assumption of a global, isotropic escape fraction and a universal MFP at each redshift.

\subsection{Lya Absorption and the Ionizing Background}

Our simulation specifies (as a function of position) the density and the peculiar velocity of the gas as well as the ionizing radiation field. We construct mock Ly $\alpha$-Forest spectra as described in Hui, Gnedin \& Zhang (1997) and briefly outline only the main points here, referring the reader to the original paper for details. Along a given sight-line, over a distance between $\boldsymbol{x}_{\mathrm{A}}$ and $\boldsymbol{x}_{\mathrm{B}}$, Hui et al. (1997) compute the optical depth as a function of observed frequency $\tau\left(v_{\mathrm{o}}\right)$ as.

$$
\tau\left(v_{o}\right)=\int_{\boldsymbol{x}_{\mathrm{A}}}^{\boldsymbol{x}_{\mathrm{B}}} \frac{\mathrm{d} x}{1+\bar{z}} n_{\mathrm{HI}}(\boldsymbol{x}) \sigma_{\alpha} .
$$

Here $\bar{z}$ is the redshift at the center of the 1-D spatial interval $\boldsymbol{x}_{\mathrm{B}}-\boldsymbol{x}_{\mathrm{A}}$ in which $\mathrm{d} x$ is the distance between cells, $\sigma_{\alpha}$ is the Ly $\alpha$ cross-section and $n_{\mathrm{HI}}(\boldsymbol{x})$ is the number density of neutral hydrogen. We consider sight-lines parallel to the edges of the simulation box in which the perpendicular, central plane of the box corresponds to $\bar{z}$, which is also the redshift of our simulation snap-shot.

The Ly $\alpha$ cross-section is expressed as a function of the velocities $u_{0}$ and $u$ which can be interpreted in terms of the observed, the intrinsic and the resonant Ly $\alpha$ frequencies

$$
\sigma_{\alpha}=\sigma_{\alpha, 0} \frac{c}{b \sqrt{\pi}} e^{-\left(u-u_{0}\right)^{2} / b^{2}},
$$

where $\sigma_{\alpha, 0}=4.5 \times 10^{-18} \mathrm{~cm}^{2}$ and $c$ is the speed of light. The parameter $b=\left(2 k_{\mathrm{B}} T / m_{\mathrm{p}}\right)^{1 / 2}$ is a function of the gas temperature $(T)$ which accounts for the thermal broadening of the line profile. Here $k_{\mathrm{B}}$ is the Boltzmann constant and $m_{\mathrm{p}}$ is the mass of a proton. Hui et al. (1997) find that the IGM gas temperature can be described locally by $T=T_{0}\left(1+\delta_{\mathrm{b}}\right)^{\gamma-1}$, with values of $1.2<\gamma<1.7$ (here $\delta_{\mathrm{b}}$ is the over-density of baryons). However, Lai et al. (2006) find the impact of temperature fluctuations on Ly $\alpha$-Forest spectra is small, therefore we assume $T_{0}=1.7 \times 10^{4} \mathrm{~K}$ and an isothermal IGM $(\gamma=1)$. We note that the uncertainty introduced by neglecting temperature fluctuations is absorbed into the uncertainty of our estimated value for the required ionization rate. Along a sight-line, $u_{0}$ is determined in each pixel by the redshift relative to $\bar{z}$ and the corresponding velocity acquired due to the Hubble flow. The parameter $u$ is determined by the peculiar velocity and the redshifted velocity of the Ly $\alpha$ resonance.

As a function of position we compute the proper number density of neutral hydrogen as

$$
n_{\mathrm{HI}}(\boldsymbol{x})=f_{n_{\mathrm{HI}}}(\boldsymbol{x}) n_{\mathrm{H}}(\boldsymbol{x}),
$$

where $f_{n_{\mathrm{HI}}}(\boldsymbol{x})$ is the neutral fraction and the density of hydrogen is given by $n_{\mathrm{H}}(\boldsymbol{x})=\bar{n}_{\mathrm{H}} \Delta(\boldsymbol{x})$. The relative over-density $\Delta(\boldsymbol{x}) \equiv \rho(\boldsymbol{x}) / \bar{\rho}$ is obtained from the $N$-body simulation and $\bar{n}_{\mathrm{H}}$ is the spatially averaged number density of hydrogen. We compute the neutral fraction (following Hui et al. 1997) as

$$
\begin{aligned}
f_{n_{\mathrm{HI}}}(\boldsymbol{x}) \sim & 1.6 \times 10^{-6}\left(\frac{T}{10^{4} \mathrm{~K}}\right)^{-0.7}\left(\frac{\Omega_{\mathrm{B}} h^{2}}{0.0125}\right) \\
& \times\left(\frac{J_{\mathrm{HI}}(\boldsymbol{x})}{0.5}\right)^{-1} \Delta(\boldsymbol{x})\left(\frac{1+\bar{z}}{4}\right)^{3} .
\end{aligned}
$$

In Equation (9) the quantity $J_{\mathrm{HI}}(\boldsymbol{x})$ is the average flux of ionizing photons in the ionizing background at wavelengths below the Lyman limit given by

$$
J_{\mathrm{HI}}(\boldsymbol{x})=\frac{\int_{\nu_{\mathrm{L}}}^{\infty} \mathrm{d} v 4 \pi J_{v} \sigma_{v} / h_{\mathrm{p}} v}{\int_{v_{\mathrm{L}}}^{\infty} \mathrm{d} v 4 \pi \sigma_{v} / h_{\mathrm{p}} v},
$$

where $J_{v}$ is the specific intensity as a function of frequency and $\sigma_{v}$ is the HI photo-ionization cross section. For the spectrum of star-burst galaxies employed in this paper (Section 2.2), the ionizing flux can be expressed relative to the specific intensity at the Lyman limit as $J_{\mathrm{HI}}=0.7 J_{21}$, which we computed in Equation (5). We note that the numerator in Equation (10) is just the ionization rate, Equation (11), highlighting the proportionality between the ionizing flux $\left(J_{\mathrm{HI}}\right)$, the ionization rate $\left(\Gamma_{-12}\right)$ and the flux at the Lyman limit $\left(J_{21}\right)$ via the SED.

We note that as it enters into the calculation of the optical depth, Equations (6)-(10), $f_{\text {esc }}$ strictly implies the escape fraction of all ionizing photons. Recent calculations of the frequency dependence of the escape fraction (Gnedin et al. 2008) show that $f_{\text {esc }}$ increases by a factor of (less than) 2 between the Hi $(13.6 \mathrm{eV})$ and HeII $(54 \mathrm{eV})$ ionization thresholds. This suggests that interpreting $f_{\text {esc }}$ as the escape fraction at the Lyman limit, leads to a small over-estimate of the quantity which is constrained via observations of galaxies as summarized in Section 1. However, the escape fraction at frequencies above the Lyman limit are weighted by a factor proportional to the adopted SED. Therefore, we expect the difference between $f_{\text {esc }}$ at the Lyman limit and the escape fraction which is explicitly constrained by our model to be small in comparison to other uncertainties in our model.

The transmission at a particular frequency can be related to the optical depth by $F\left(v_{0}\right)=\exp \left[-\tau\left(v_{0}\right)\right]$. Using the model for computing the optical depth presented above, we construct Ly $\alpha$-Forest spectra by computing the transmission over a range of frequencies $\left(v_{\mathrm{o}}\right)$. Our approach is to generate an ensemble of 3072 absorption spectra for evenly spaced sight-lines throughout our simulation box. Along each sight-line we then compute the effective transmission $\left(F_{\text {eff }}\right)$ over stretches of the forest which enables direct comparison with the observational data. From the ensemble of $F_{\text {eff }}$ we calculate the mean effective transmission, $\bar{F}_{\text {eff }}$. Finally, we rescale the optical depth in each pixel (via $f_{\text {esc }}$ ) so that our simulated value of $\bar{F}_{\text {eff }}$ matches a particular value. 


\subsection{The Observed Transmission of the IGM and the MFP}

For a sample of 19 Ly $\alpha$ absorption spectra observed towards high redshift quasars, Fan et al. (2006) measure the average (or effective) transmission over a wavelength range in the Ly $\alpha$ Forest which corresponds to a redshift interval of $\Delta z=0.15$. From these transmission measurements along the available sight-lines, Fan et al. (2006) compute the mean effective transmission $\left(\bar{F}_{\text {eff }}^{\text {obs }}\right)$ in redshift slices (of thickness 0.2) of the IGM, which are centered on slightly different redshifts to the redshifts of our simulations $(\bar{z})$. We therefore estimate values of $\bar{F}_{\text {eff }}^{\text {obs }}$ at $\bar{z}=5.5$, 5.7, 6.0 directly from the measurements of $F_{\text {eff from Fan }}$ et al. (2006) (their Table 2) within redshift bins of 0.2 centered on each $\bar{z}$. We compute the mean and the variance in this sample assuming a Gaussian distribution for uncertainties on individual transmission measurements. At $z=5.5,5.7,6.0$ the observed mean effective transmission values (and $1-\sigma$ scatter) are $\bar{F}_{\text {eff }}^{\text {obs }}=0.079 \pm 0.035$, $0.047 \pm 0.031,0.006 \pm 0.005$.

At $z=5.5,5.7,6.0$, we take values of the MFP estimated by Fan et al. (2006), which assume a frequency averaged ionization cross-section, $\left\langle\sigma_{v}\right\rangle$, corresponding to an ionizing background spectrum defined by $J_{v} \propto v^{-5}$. We re-compute $\left\langle\sigma_{\nu}\right\rangle$ using the spectrum appropriate for our model and adjust the values for $\lambda_{\mathrm{mfp}}$ accordingly. At the above redshifts we consider the MFPs, $\lambda_{\mathrm{mfp}}=28.7$, 18.9, 4.2 Mpc/h respectively. Although these MFPs are derived from observational data, we note that they are model dependent and assume a uniform ionizing background. We discuss the uncertainty in the MFP further in Section 3.2.

\section{The Ionization Rate and the Escape Fraction}

In this section we present our estimates of the Hi ionization rate $(\Gamma)$ and the escape fraction of ionizing photons $\left(f_{\text {esc }}\right)$, which we have constrained by adjusting the level of the ionizing background in our model so as to reproduce the transmission measurements $\left(\bar{F}_{\text {eff }}^{\text {obs }}\right)$ inferred from the spectra of high redshift quasars (Fan et al. (2006).

\subsection{Constraints on the Ionization Rate}

The Hr ionization rate is computed from the flux in the ionizing background $\left(J_{v}\right)$ at all frequencies above the Lyman $\operatorname{limit}\left(v_{\mathrm{L}}\right)$ by

$$
\Gamma=4 \pi \int_{\nu_{\mathrm{L}}}^{\infty} \frac{J_{v}}{h_{\mathrm{p}} v} \sigma_{\nu} \mathrm{d} \nu,
$$

where $\sigma_{v}$ is the HI photo-ionization cross-section, and $h_{\mathrm{p}}$ is Planck's constant. For our spectrum of an ionizing background due to star-burst galaxies this relationship can be expressed as $\Gamma_{-12}=2.8 J_{21}$, where $\Gamma_{-12}$ is the photo-ionization rate in units of $10^{-12} \mathrm{~s}^{-1}$. To find the ionization rate in the IGM from the Ly $\alpha$-Forest spectra we trial values of $f_{\text {esc }}$, which is the free parameter we use to scale the intensity of the ionizing background. For each $f_{\text {esc }}$, we use Equation (5) to find $J_{21}$, and hence



Figure 2 Our estimates of the $\mathrm{HI}$ ionization rates at $z=5.5,5.7,6.0$ assuming MFPs $\lambda_{\text {mfp }}=28.7,18.9,4.2 \mathrm{Mpc} / \mathrm{h}$ and mean transmission values of $\bar{F}_{\text {eff }}=0.079,0.047,0.006$, respectively (open circles). For comparison we show estimates of $\Gamma_{-12}$ from Bolton \& Haehnelt (2007, diamonds) and Fan et al. (2006, points) which are also based on IGM Ly $\alpha$ transmission. We also show our constrained values of $\Gamma_{-12}$ assuming uniform ionizing backgrounds at each redshift (open squares).

the neutral fraction via Equation (9). We compute Ly $\alpha$ Forest spectra from the absorption at a range of frequencies computed via Equation (6). A value for the effective optical depth is computed from the Ly $\alpha$-Forest spectra along many sight-lines for a given $f_{\text {esc }}$. The value of $f_{\text {esc }}$ is then adjusted until the simulation reproduces the observed, mean Ly $\alpha$ absorption.

In Figure 2 we show the mean ionization rates constrained using our model for both a non-uniform background (open-circles) and a uniform background. We note that in our model the ionization rate is spatially non-uniform as it follows the non-uniform ionizing background and so our estimates of the mean ionization rate are explicitly $\left\langle\Gamma_{-12}(\boldsymbol{x})\right\rangle$. We compare our results to the ionization rates predicted by Bolton \& Haehnelt (2007, diamonds) and Fan et al. (2006, points). The 1- $\sigma$ error in the ionization rate reflects the 1- $\sigma$ uncertainty in the observed transmission at each redshift. Our results are in excellent agreement with Bolton \& Haehnelt (2007) and a factor of $\sim 2$ greater than those of Fan et al. (2006). We refer the reader to the discussions in Bolton et al. (2005) and Bolton \& Haehnelt (2007) regarding the likely under-estimation of the ionization rates predicted by the fluctuating Gunn-Peterson approximation model assumed by Fan et al. (2006). It is also worth noting that our values for $\Gamma_{-12}$ assuming a uniform background are less than those for a non-uniform background, in agreement with the findings of previous authors (Gnedin \& Hamilton 2002; Meiksin \& White 2004; Croft 2004; Bolton \& Haehnelt 2007). In our model for a fluctuating ionizing background, high ionization rates are biased to overdense regions and the transmission of rare voids remains largely unaffected by increasing the level of the ionizing 


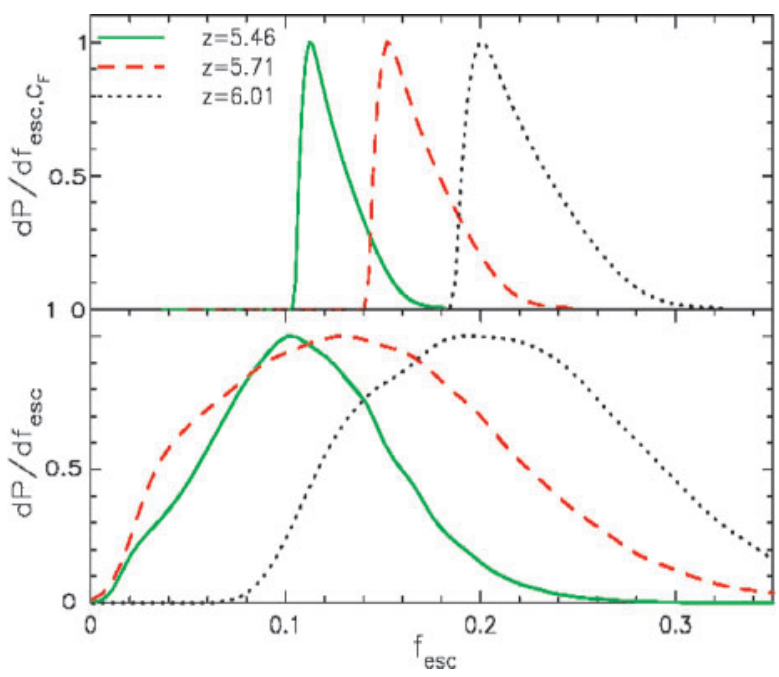

Figure 3 The likelihood distribution $\mathrm{d} P / \mathrm{d} f_{\text {esc }}$. We have adjusted the intensity of the ionizing background such that the model values for the mean transmission match the observed values. At $z=5.5$, 5.7, 6.0, these mean transmission values are $\bar{F}_{\text {eff }}=0.079,0.047$, 0.006 , respectively. The ionizing background model is defined by assumed MFPs of $\lambda_{\mathrm{mfp}}=28.7,18.9,4.2 \mathrm{Mpc} / h$ respectively. The upper panel shows the distributions of $f_{\text {esc }}$ corresponding to the mean transmission. The lower panel shows the distributions of $f_{\mathrm{esc}}$ after integration over the uncertainty in the mean transmission.

background. In contrast, raising the level of a uniform ionizing background changes the transmission of all regions (see Bolton \& Haehnelt 2007 and references therein). On the other hand, the contribution of low density regions to the aggregate Ly $\alpha$ absorption observed along a LOS is relatively minor, irrespective of the ionizing background.

\subsection{The Escape Fraction of Ionizing Photons}

In our model, we adjust the intensity of the ionizing background (via the parameter $f_{\text {esc }}$ ) in order to reproduce a given effective transmission $(F)$. Following this constraint of the escape fraction which corresponds to a particular $F$, we calculate the product $\mathcal{C}_{\mathrm{F}}=f^{\star} f_{\text {esc }}$. Our previous constraint of the SFR via the parameters $f^{\star}$ and $t_{\mathrm{DC}}$ (see Section 2.2) describes the distribution $\mathrm{d} P / \mathrm{d} t_{\mathrm{DC}} \mathrm{d} f^{\star}$. From this distribution we then form the distribution $\mathrm{d} P / \mathrm{d} f^{\star}$ by marginalizing over $t_{\mathrm{DC}}$. Given $\mathrm{d} P / \mathrm{d} f^{\star}$, we compute the probability distribution of $f_{\text {esc }}$ for a particular transmission $\left(\mathrm{d} P /\left.\mathrm{d} f_{\mathrm{esc}}\right|_{\mathcal{C}_{\mathrm{F}}}\right)$ using

$$
\left.\frac{\mathrm{d} P}{\mathrm{~d} f_{\text {esc }}}\right|_{\mathcal{C}_{\mathrm{F}}}=\frac{\mathrm{d} P}{\mathrm{~d} f^{\star}}\left|\frac{\mathrm{d} f^{\star}}{\mathrm{d} f_{\mathrm{esc}}}\right|_{\mathcal{C}_{\mathrm{F}}}=\frac{\mathrm{d} P}{\mathrm{~d} f^{\star}} \frac{\mathcal{C}_{\mathrm{F}}}{f_{\text {esc }}^{2}} .
$$

The upper panel in Figure 3 shows the probability distributions of $\mathrm{d} P /\left.\mathrm{d} f_{\mathrm{esc}}\right|_{\mathcal{C}_{\mathrm{F}}}$ at $z=5.5,5.7,6.0$ assuming ionizing backgrounds in which the MFPs of ionizing photons are, $\lambda_{\mathrm{mfp}}=28.7,18.9,4.2 \mathrm{Mpc} / \mathrm{h}$. In this figure $\mathcal{C}_{\mathrm{F}}$ corresponds to the observed mean effective transmission (i.e $F=\bar{F}_{\text {eff }}$ ). Our analysis suggests $f_{\text {esc }} \sim 10-25 \%$ over the redshift range $z \sim 5.5-6$. Theses distributions represent the statistical uncertainty in $f_{\text {esc }}$ owing to uncertainty in the modelling of the LF.
The lower panel in Figure 3 shows the probability distributions of $f_{\text {esc }}$ including the additional uncertainty owing to measurement error in the transmission. To find this distribution,

$$
\left.\frac{\mathrm{d} P}{\mathrm{~d} f_{\text {esc }}} \propto \int \mathrm{d} \mathcal{C}_{\mathrm{F}} \frac{\mathrm{d} P}{\mathrm{~d} f_{\text {esc }}}\right|_{\mathcal{C}_{\mathrm{F}}} \frac{\mathrm{d} P}{\mathrm{~d} F} \frac{\mathrm{d} F}{\mathrm{~d} \mathcal{C}_{\mathrm{F}}},
$$

we assume the observed transmission to follow a Gaussian distribution $(\mathrm{d} P / \mathrm{d} F)$ with values for the mean and variance that are given in Section 2.4. The distributions, $\mathrm{d} P /\left.\mathrm{d} f_{\text {esc }}\right|_{\mathcal{C}_{\mathrm{F}}}$, can be evaluated from Equation (12), and $\mathrm{d} F / \mathrm{d} \mathcal{C}_{\mathrm{F}}$ is computed from the simulations. The uncertainty in transmission dominates the uncertainty in estimates of $f_{\text {esc }}$. We find a statistical relative error on the value of $f_{\text {esc }}$ that is of order $\sim 50 \%$, which is larger than the difference between the most likely values at each redshift.

The value of the MFP warrants further discussion. The MFPs assumed in our model are based on those derived in Fan et al. (2006) which are generally lower (by a factor of $\gtrsim 2)$ than those suggested by Bolton \& Haehnelt (2007). The MFP estimates of Bolton \& Haehnelt (2007) assume that photons propagate freely in regions below some critical over-density which may be determined from the ionization rate and the column density $\left(N_{\mathrm{HI}}\right)$ of selfshielded neutral clouds. In contrast, Fan et al. (2006) estimate the MFP considering the distribution of the neutral fraction over all over-densities in the IGM. Although our estimates of the ionization rate are in agreement with Bolton \& Haehnelt (2007), we choose to adopt lower values for the MFPs for two reasons. Firstly, the MFP of ionizing photons may be over-estimated by a factor of $\sim 2$ by neglecting Hi absorbers between Lyman-limit systems (Miralda-Escudé 2003; Furlanetto \& Oh 2005). Secondly, as the MFP is very uncertain, adopting the lower values is conservative with respect to the photon budget for reionization as we will discuss in Section 7.

We note that uncertainty in the MFP represents a large source of uncertainty in our estimates of the escape fraction, and we therefore next investigate the sensitivity of the results to the MFP. In Figure 4 we plot the distribution of the escape fraction (upper panel) corresponding to the observed mean transmission at $z=5.5$, assuming several MFPs $(3.5,7.0,18.9,28.7,70.0 \mathrm{Mpc} / h)$. An increased MFP leads to a decrease in the escape fraction required to reproduce the same ionizing background. For the shortest (longest) MFP considered $\lambda=3.5$ (70.0) Mpc/ $h$ the most likely value for the escape fraction is $f_{\text {esc }} \sim 10.2$ $(1.3 \%)$. This range represents a factor of $\sim 10$ variation in the escape fraction given a range of a factor of $\sim 20$ in MFP. In the lower panel we plot the distribution of $\mathrm{d} P / \mathrm{d}\left(\lambda f_{\text {esc }}\right)$, where

$$
\frac{\mathrm{d} P}{\mathrm{~d}\left(\lambda_{\mathrm{mfp}} f_{\mathrm{esc}}\right)}=\frac{\mathrm{d} P}{\mathrm{~d} f^{\star}} \frac{\mathcal{C}_{\mathrm{F}}}{\lambda_{\mathrm{mfp}} f_{\mathrm{esc}}^{2}},
$$

where $\mathcal{C}_{\mathrm{F}}$ corresponds to the mean observed transmission. This demonstrates the level of degeneracy of the product $\lambda_{\mathrm{mfp}} f_{\mathrm{esc}}$. For the shortest (longest) MFP considered $\lambda_{\mathrm{mfp}}=3.5(70.0) \mathrm{Mpc} / h$ the most likely value in the 



Figure 4 Likelihood distributions for $f_{\mathrm{esc}}$ and $\left(\lambda f_{\mathrm{esc}}\right)$ corresponding to the observed mean transmission at $z=5.5$ for ionizing backgrounds defined by MFPs 3.5, 7.0, 18.9, 28.7, 70.0 Mpc/h. We have adjusted the intensity of the ionizing background such that the model values for the mean transmission match the observed value. At $z=5.5$, the observed mean transmission is $\bar{F}_{\text {eff }}=0.079$. In the upper panel the maximum likelihood occurs for $f_{\text {esc }}=70,30,13,11$, $9 \%$ respectively. In the lower panel the maximum likelihood occurs for $\lambda_{\mathrm{mfp}} f_{\mathrm{esc}}=2.4,2.1,2.5,3.2,6.3 \mathrm{Mpc} / \mathrm{h}$ respectively.

product $\lambda_{\text {mfp }} f_{\text {esc }}$ is $\sim 2.4(6.3) \mathrm{Mpc} / h$. This represents a factor of less than 3 . Thus we can summarize our results with the relation $f_{\text {esc }}\left(\lambda_{\text {mfp }}\right) \sim K_{\lambda}\left(\lambda_{\text {mfp }} / \mathrm{Mpc} / h\right)^{-1}$, where $2 \lesssim K_{\lambda} \lesssim 6$. Excluding our estimate of $f_{\text {esc }}$ at the longest MFP this range for $K_{\lambda}$ narrows to $2 \lesssim K_{\lambda} \lesssim 3$.

\subsection{Further Discussion}

Before proceeding it is illustrative to compare estimates of the escape fraction which are derived by (Bolton \& Haehnelt 2007). Bolton \& Haehnelt (2007) find the escape fraction to be $20-30 \%$ by comparing the constrained ionization rate to the global emissivity, which they compute by integrating the observed UV luminosity function (LF) down to a minimum galaxy brightness of $M_{1350}=-18$. They also consider quasars to contribute to the global emissivity, however this contribution is less than $\sim 10 \%$ and not considered further here. In our model we include sources down to a lower luminosity $\left(M_{1350} \sim-16\right)$ than used in Bolton \& Haehnelt (2007), which corresponds to a factor of $\sim 2$ difference in the integrated emissivity. However this factor is partially cancelled by including the revised galaxy LF of Bouwens et al. (2007), which leads to a decrease in the integrated emissivity of a factor of $\sim 2$. In addition, the SED assumed in our study is $\sim 35 \%$ harder (Bolton \& Haehnelt 2007 use $\left.\Gamma_{-12}=2.1 J_{21}\right)$. Finally, the definition of the escape fraction used in Bolton \& Haehnelt (2007) is equivalent to the relative escape fraction which is frequently quoted in observational studies (see Section 1). This is because they infer the escape fraction of ionizing photons having assumed an emissivity which is normalized by integrating over the observed LF of Lyman break galaxies. Following Bouwens et al. (2007), this emissivity should be corrected by a factor of 1.5 to describe the intrinsic emission of galaxies (at $1350 \AA$ ) at $z \sim 6$. An important point of difference between our work and Bolton \& Haehnelt (2007) is that the identification of halos as the sources allows the escape fraction to be connected with halo mass as well as luminosity, rather than luminosity alone.

The above corrections should be considered in order to quantitatively compare our estimate of $f_{\text {esc }}$ to that of Bolton \& Haehnelt (2007). Following these corrections the result of Bolton \& Haehnelt (2007) becomes $f_{\text {esc }} \sim 15-25 \%$, which is consistent with our estimates. Although both studies are normalized to the observed LF, a significant distinction in our model is that the ionizing background is produced by discrete sources and so spatially non-uniform. However, the consistency of our results with Bolton \& Haehnelt (2007) suggests that distinction between a global escape fraction derived by comparing to the integrated emissivity, and a globally averaged $f_{\text {esc }}$ which is apriori attributed to individual galaxies, is not significant.

Finally, we note an additional source of uncertainty in our model which is associated with the assumed isotropic escape of ionizing radiation from galaxies. Previous studies (Fujita et al. 2003; Razoumov \& Sommer-Larsen 2006; Gnedin et al. 2008) have shown that ionizing radiation escapes from host galaxies through ionized tunnels in the interstellar medium created by radiative feedback. This may account for the range of values derived for $f_{\text {esc }}$ from observation of galaxies along different sight-lines. In our model, this anisotropy would increase the fluctuations in the ionizing background. Based on our finding that the impact of a non-uniform ionizing background on the global value of $f_{\text {esc }}$ is not significant, we expect that the uncertainty introduced to the value of $f_{\text {esc }}$ by the assumption of an isotropic escape fraction in our model is not significant.

\section{Suppression of Dwarf Galaxies}

In the previous section we considered an ionizing background in which we included all halos down to the mass resolution of the simulation $\left(2 \times 10^{9} M_{\odot}\right)$. In our best fit model this mass corresponds to a luminosity of $M_{1350} \sim-16$. In this section we investigate the impact on the inferred escape fraction of allowing the suppression of dwarf galaxies. The reionization of the IGM elevates the Jeans mass and so increases the required minimum virial temperature a DM halo must have in order for gas to accrete and form stars. This minimum virial temperature could be as large as $\sim 2.5 \times 10^{5} \mathrm{~K}$ which corresponds to a minimum halo mass, $M_{\mathrm{vir}} \sim 10^{10} \mathrm{M}_{\odot}$ (Efstathiou 1992; Thoul \& Weinberg 1996; Dijkstra et al. 2004). The suppression of small galaxies will modify both the topology and the level of the ionizing background and hence also 




Figure 5 The best fit model LF with parameter values of $t_{\mathrm{DC}}=0.18$ $\left(t_{\mathrm{s}}=2.5 \times 10^{8} \mathrm{yr}\right)$ and $f^{*}=0.12$, as constrained by a maximum likelihood analysis. Inset is is a plot of the likelihood distribution in which the contours represent the 1- $\sigma, 2-\sigma$ and 3- $\sigma$ confidence levels with a cross to mark the point of maximum likelihood. For display purposes a line joins the model points (solid). The error bars on the model reflect the number of galaxies in each luminosity bin as measured from our simulation. The open circles represent the data from the observed UV galaxy LF (Bouwens et al. 2007). The model points are shifted slightly to the right of the observational data for clarity.

the derived estimate of the escape fraction. We repeat our analysis of the galaxy LF, limiting the mass of halos which are considered as potential sources of UV radiation to $M>10^{10} \mathrm{M}_{\odot}$.

Repeating our analysis of Section 2.2, Figure 5 shows the best fit LF for the suppression of galaxies with halo mass less than $10^{10} \mathrm{M}_{\odot}$. For display purposes a line joins the model points (solid) which are the centers of the constructed luminosity bins. At the faint end of the LF we have constructed an additional bin as we are able to resolve galaxies down to a lower luminosity in our simulation than are currently observed. The error bars on the model reflect the number of galaxies in each luminosity bin. The open circles represent the data from the observed UV galaxy LF (Bouwens et al. 2007). The model points are shifted slightly to the right of the observational data for clarity only. Inset in Figure 5 is a plot of contours representing the 1- $\sigma, 2-\sigma$ and 3- $\sigma$ confidence levels on the parameters $f^{\star}$ and $t_{\mathrm{DC}}$. A cross marks the point of maximum likelihood, with values of $t_{\mathrm{DC}}=0.18\left(t_{\mathrm{s}}=2.5 \times 10^{8} \mathrm{yr}\right)$ and $f^{*}=0.12$. These values are not significantly different from those previously derived for the inclusion of galaxies with halo mass down to $10^{9} \mathrm{M}_{\odot}$ (Section 2.2). For the suppression of galaxies with halo mass less than $10^{10} \mathrm{M}_{\odot}$, the minimum mass halo which contributes to the ionizing background corresponds to a specific luminosity of $L \sim 4.3 \times 10^{27} \mathrm{erg} \mathrm{s}^{-1} \mathrm{~Hz}^{-1}$ and a magnitude of $M_{1350} \sim-17.5$. This luminosity is smaller than the

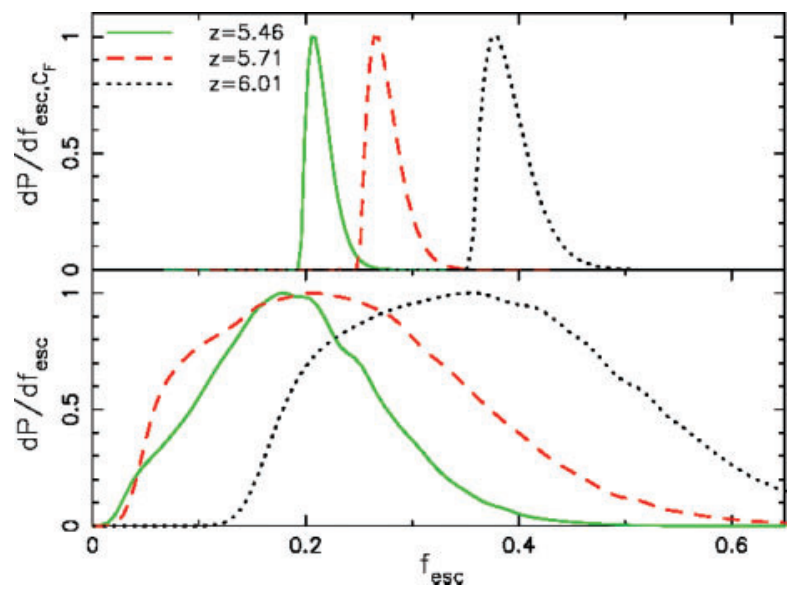

Figure 6 The likelihood distribution $\mathrm{d} P / \mathrm{d} f_{\text {esc }}$. We have adjusted the intensity of the ionizing background such that the model values for the mean transmission match the observed values. At $z=5.5$, 5.7, 6.0, these mean transmission values are $\bar{F}_{\text {eff }}=0.079,0.047$, 0.006 , respectively. The ionizing background model is defined by assumed MFPs of $\lambda_{\mathrm{mfp}}=28.7,18.9,4.2 \mathrm{Mpc} / h$ respectively. The upper panel shows the distributions of $f_{\text {esc }}$ corresponding to the mean transmission. The lower panel shows the distributions of $f_{\text {esc }}$ after integration over the uncertainty in the mean transmission.

minimum luminosity included in Bolton \& Haehnelt (2007)

Finally, we compute the likelihood distributions for the escape fraction, analogous to those shown in Figure 3. In the upper panel of Figure 6 we show the probability distributions of $f_{\text {esc }}$ at our fiducial redshifts and MFPs, where we have constrained the transmission to the mean observed transmission. This yields values for $f_{\text {esc }}$ between $20 \%$ and $40 \%$, which are larger than those found in Figure 3, where we included sources down to a halo mass of $\sim 10^{9} \mathrm{M}_{\odot}$. The distributions in the upper panel of Figure 6 represent uncertainty in $f_{\text {esc }}$ owing to uncertainty in the modelling of the LF. The lower panel in Figure 6 shows the probability distributions of $f_{\text {esc }}$ for the same redshifts and MFPs as the lower panel, however here we have included the additional uncertainty introduced by the distribution of optical depth measurements. As before, we assume the observed transmission to follow a Gaussian distribution $(\mathrm{d} P / \mathrm{d} F)$ with values for the mean and variance that are given in Section 2.4.

\section{Reionization by Massive Galaxies}

In a recent paper, Gnedin et al. (2008) suggested that the escape fraction of ionizing photons is a few percent for massive galaxies, but is negligibly small for galaxies which reside in halos of mass $M \lesssim 5 \times 10^{10} \mathrm{M}_{\odot}$. We investigate the implications for the escape fraction inferred from Ly $\alpha$ transmission data by excluding halos of mass $M<5 \times 10^{10} \mathrm{M}_{\odot}$. The suggestion that ionizing photons do not escape galaxies with halo mass less than $5 \times 10^{10} \mathrm{M}_{\odot}$ does not imply that photons above the Lyman limit (i.e. $1350 \AA$ ) are similarly affected. Therefore we assume the values for $f^{\star}$ and $t_{\mathrm{DC}}$ from Section 4 to compute the ionizing background in this case. In this 


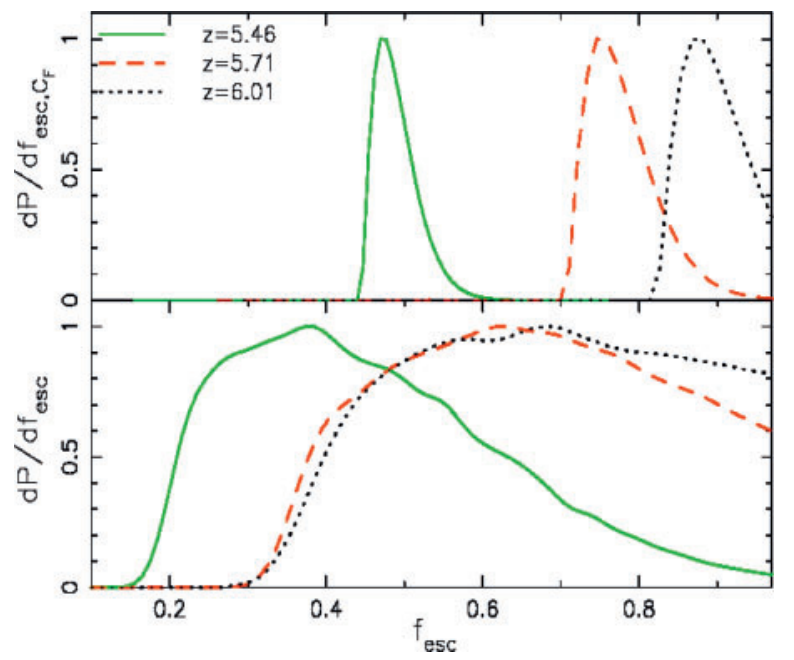

Figure 7 The likelihood distribution $\mathrm{d} P / \mathrm{d} f_{\text {esc }}$. We have adjusted the intensity of the ionizing background such that the model values for the mean transmission match the observed values. At $z=5.5$, 5.7, 6.0, these mean transmission values are $\bar{F}_{\text {eff }}=0.079,0.047$, 0.006 , respectively. The ionizing background model is defined by assumed MFPs of $\lambda_{\mathrm{mfp}}=28.7,18.9,4.2 \mathrm{Mpc} / h$ respectively. The upper panel shows the distributions of $f_{\text {esc }}$ corresponding to the mean transmission. The lower panel shows the distributions of $f_{\text {esc }}$ after integration over the uncertainty in the mean transmission.

case, the minimum halo mass corresponds to a minimum specific luminosity of $L \sim 2.2 \times 10^{28} \mathrm{erg} \mathrm{s}^{-1} \mathrm{~Hz}^{-1}$ and a magnitude of $M_{1350} \sim-19.3$, which is brighter than the minimum magnitude considered by Bolton \& Haehnelt (2007).

Again we compute the likelihood distributions for the escape fraction. In the upper panel of Figure 7 we show the probability distributions of $f_{\text {esc }}$ at our fiducial redshifts and MFPs, where we have constrained the transmission to the mean observed transmission. This yields values for $f_{\text {esc }}$ between 46 and $86 \%$, which are larger again than those found in Figure 3, where we included sources down to a halo mass of $\sim 10^{9} \mathrm{M}_{\odot}$. As previously computed in Section 3.2, the lower panel in Figure 7 shows the distributions of $f_{\text {esc }}$ in which we have included the uncertainty introduced by the distribution of optical depth measurements at each redshift. These results imply that reionization by massive galaxies alone, with escape fractions of order a few percent (Gnedin et al. 2008) is not consistent with the measured ionization rate at $z \sim 5-6$.

\section{Extrapolation of Numerical Results to Other Galaxy Masses}

In Figure 8 we summarize our estimates of the escape fraction for the three minimum mass scenarios we have considered. The numerical estimates are shown including the range for the median value of $f_{\text {esc }}$ predicted in our simulation at the three redshifts modelled. For comparison, the dashed lines represent simple analytic fits to describe the relationship between the minimum mass of halos which contribute to the ionizing background and the escape fraction. To estimate this relationship we assume that the product of the escape fraction and the star-formation rate



Figure 8 Extrapolation of estimates of $f_{\text {esc }}$ as a function of minimum mass from numerical simulations. Dashed lines show analytic fits to the numerical data for both the maximum and minimum values found for the normalization parameter $A$ (see text). The numerical estimates are shown including the range for the median value of $f_{\text {esc }}$ predicted in our simulation at the three redshifts modelled. The analytic fit suggests that the value for $f_{\text {esc }}$ is greater than $100 \%$ for minimum masses, $M_{\min } \gtrsim 10^{12}$, and approaches a constant value of $\sim 5-10 \%$ for minimum masses near the hydrogen cooling threshold. Figure 8 indicates that the escape fraction from high redshift galaxies must be $\gtrsim 5 \%$ irrespective of the minimum mass.

is constant and that the star-formation rate can be approximated as being proportional to the time derivative of the collapsed mass fraction, $\mathrm{d} F_{\mathrm{col}} / \mathrm{d} t$. The quantity $\mathrm{d} F_{\mathrm{col}} / \mathrm{d} t$ is computed analytically as a function of halo mass and redshift using the prescription of (Sheth \& Tormen 1999). Thus the escape fraction can be expressed as

$$
f_{\mathrm{esc}}\left(M_{\min }\right)=A\left(M_{\min }\right)\left[\frac{\mathrm{d} F_{\mathrm{col}}}{\mathrm{d} t}\left(M_{\mathrm{min}}\right)\right]^{-1},
$$

where $M_{\min }$ is the minimum halo mass of galaxies which contribute to the ionizing background, and $A$ is a constant.

We normalize the relation between the escape fraction and the star-formation rate using our estimates of $f_{\text {esc }}$ from the simulations (at $z=5.5,5.7,6.0$ ), in which the minimum contributing halo masses $\left(M=2 \times 10^{9}, 10^{10}\right.$, $\left.5 \times 10^{10} \mathrm{M}_{\odot}\right)$ are adopted to compute $\mathrm{d} F / \mathrm{d} t$. The analytic time derivative of the collapsed mass faction $\left(\mathrm{d} F_{\mathrm{col}} / \mathrm{d} t\right)$ does not perfectly describe the SFR from the simulation. The value of $A$ is therefore mass and redshift dependent and the relative variation in this normalization parameter is found to be $7 \%(11 \%)$ for minimum-mass halos of $M=2 \times 10^{9}-5 \times 10^{10}$. In Figure 8 we plot $f_{\text {esc }}\left(M_{\min }\right)$ (dashed lines) assuming both the maximum and minimum values found for $A$.

Figure 8 shows that as the minimum mass is increased, the global escape fraction increases to compensate for the lost emissivity. The analytic fit suggests that the value for $f_{\text {esc }}$ is greater than $100 \%$ for minimum masses, $M_{\text {min }} \gtrsim 10^{12}$, indicating an upper limit to $M_{\min }$. The values of $f_{\text {esc }}\left(M_{\min }\right)$ can be extrapolated to masses below the mass resolution limit of the simulation $\left(2 \times 10^{9} \mathrm{M}_{\odot}\right)$ 
as shown in Figure 8. The escape fraction approaches a constant value of $\sim 5-10 \%$ for minimum masses near the hydrogen cooling threshold. These escape fractions are comparable to observed estimates for individual galaxies at lower redshift. Interestingly, Figure 8 indicates that the escape fraction from high redshift galaxies must be $\gtrsim 5 \%$ irrespective of the minimum mass.

\section{Models of the Reionization History}

The Ly $\alpha$ absorption probes the instantaneous emissivity of the galaxy population, and hence allows the constraints on the escape fraction presented thus far. However, the redshift at which reionization occurs is dependent on the cumulative star-formation. In the remainder of this paper we discuss constraints on the reionization history.

\subsection{Semi-analytic Model for Reionization}

In this section we use a semi-analytic model to study the implications of the implied values of escape fraction on the reionization history of the IGM. Miralda-Escudé, Haehnelt \& Rees (2000) presented a formalism which allows the calculation of an effective recombination rate in an inhomogeneous universe by assuming a maximum over-density $\left(\Delta_{\mathrm{c}}\right)$ penetrated by ionizing photons within Hil regions. Their model assumes that reionization progresses rapidly through islands of lower density prior to the overlap of individual cosmological ionized regions. Following the overlap epoch, the remaining regions of high density are gradually ionized. It is therefore hypothesized that at any time, regions with gas below some critical over-density $\Delta_{\mathrm{i}} \equiv \rho_{\mathrm{i}} /\langle\rho\rangle$ are highly ionized while regions of higher density are not. In what follows, we draw primarily from their prescription and refer the reader to the original paper for a detailed discussion of its motivations and assumptions. Wyithe \& Loeb (2003) employed this prescription within a semi-analytic model of reionization. This model was extended by Srbinovsky \& Wyithe (2007) and by Bolton \& Haehnelt (2007). We refer the reader to those papers for a full description.

Within the formalism of Miralda-Escude et al. (2000) we describe the post-overlap evolution of the IGM by computing the evolution of the fraction of mass in regions with over-density below $\Delta_{i}$,

$$
F_{\mathrm{M}}\left(\Delta_{\mathrm{i}}\right)=\int_{0}^{\Delta_{\mathrm{i}}} \mathrm{d} \Delta P_{\mathrm{V}}(\Delta) \Delta,
$$

where $P_{\mathrm{V}}(\Delta)$ is the volume weighted probability distribution for $\Delta$. Miralda-Escude et al. (2000) quote a fitting function which provides a good fit to the volume weighted probability distribution for the baryon density in cosmological hydrodynamical simulations. This probability distribution remains a reasonable description at high redshift when confronted with a more modern cosmology and updated simulations, although an analytical approximation for the high density tail of the distribution remains necessary as a best guess at correcting for numerical resolution (Bolton \& Haehnelt 2007).
Prior to the overlap epoch, a constant value $\Delta_{i}=\Delta_{c}$ is assumed, while in the post overlap era our model for the reionization history computes the evolution of $\Delta_{i}$. We compute evolution of the quantity $Q_{\mathrm{i}}$ which is defined to be the volume filling factor within which all matter at densities below $\Delta_{c}$ has been ionized. Within our formalism, the epoch of overlap is precisely defined as the time when $Q_{\text {i }}$ reaches unity. However, we have only a single equation to describe the evolution of two independent quantities $Q_{\mathrm{i}}$ and $F_{\mathrm{M}}$ (or equivalently $\Delta_{\mathrm{c}}$ ). The relative growth of these depends on the LF and spatial distribution of the sources. We assume $\Delta_{c}$ to be constant with redshift before the overlap epoch and compute results for models with values of $\Delta_{c}=5$. Within the formalism of Miralda-Escude et al. (2000), a maximum over-density of $\Delta_{c}=5$ corresponds approximately to the mean separation between galaxies at high redshift. For a large range of values, the effect of varying $\Delta_{c}$ is smaller than other uncertainties in the problem (Wyithe et al. 2007).

Our approach is to compute a reionization history given a particular value of $\Delta_{c}$, combined with assumed values for the efficiency of star-formation and the fraction of ionizing photons that escape from galaxies. With this history in place we then compute the evolution of the background radiation field due to these same sources. After the overlap epoch, ionizing photons will experience attenuation due to residual over-dense pockets of HI gas. We use the description of Miralda-Escude et al. (2000) to estimate the ionizing photon mean-free-path, and subsequently derive the attenuation of ionizing photons. We then compute the flux at the Lyman limit in the IGM due to sources immediate to each epoch, in addition to redshifted contributions from earlier epochs.

As in earlier sections we assume an ionizing background dominated by galaxies and the spectral energy distribution (SED) of Population-II star forming galaxies, using the model presented in Leitherer et al. (1999). The star-formation rate per unit volume is computed based on the collapsed fraction obtained from the extended Press \& Schechter (1974) model (Bond et al. 1991) in halos above the minimum halo mass for star formation, together with an assumed star formation efficiency.

In a cold neutral IGM beyond the redshift of reionization, the collapsed fraction should be computed for halos of sufficient mass to initiate star formation. The critical virial temperature is set by the temperature $\left(T_{\mathrm{N}} \sim 10^{4} \mathrm{~K}\right)$ above which efficient atomic hydrogen cooling promotes star formation. Following the reionization of a region, the Jeans mass in the heated IGM limits accretion to halos above $T_{\mathrm{I}} \sim 10^{5} \mathrm{~K}$ (Efstathiou 1992; Thoul \& Weinberg 1996; Dijkstra et al. 2004b). As described in the introduction, only a fraction of ionizing photons produced by stars enter the IGM. Therefore an additional factor of $f_{\text {esc }}$ (the escape fraction) must be included when computing the emissivity of galaxies. In our fiducial model we assume this escape fraction to be independent of mass. We define a parameter $f_{\text {esc }}^{*} \equiv f^{\star} f_{\text {esc }}$. 

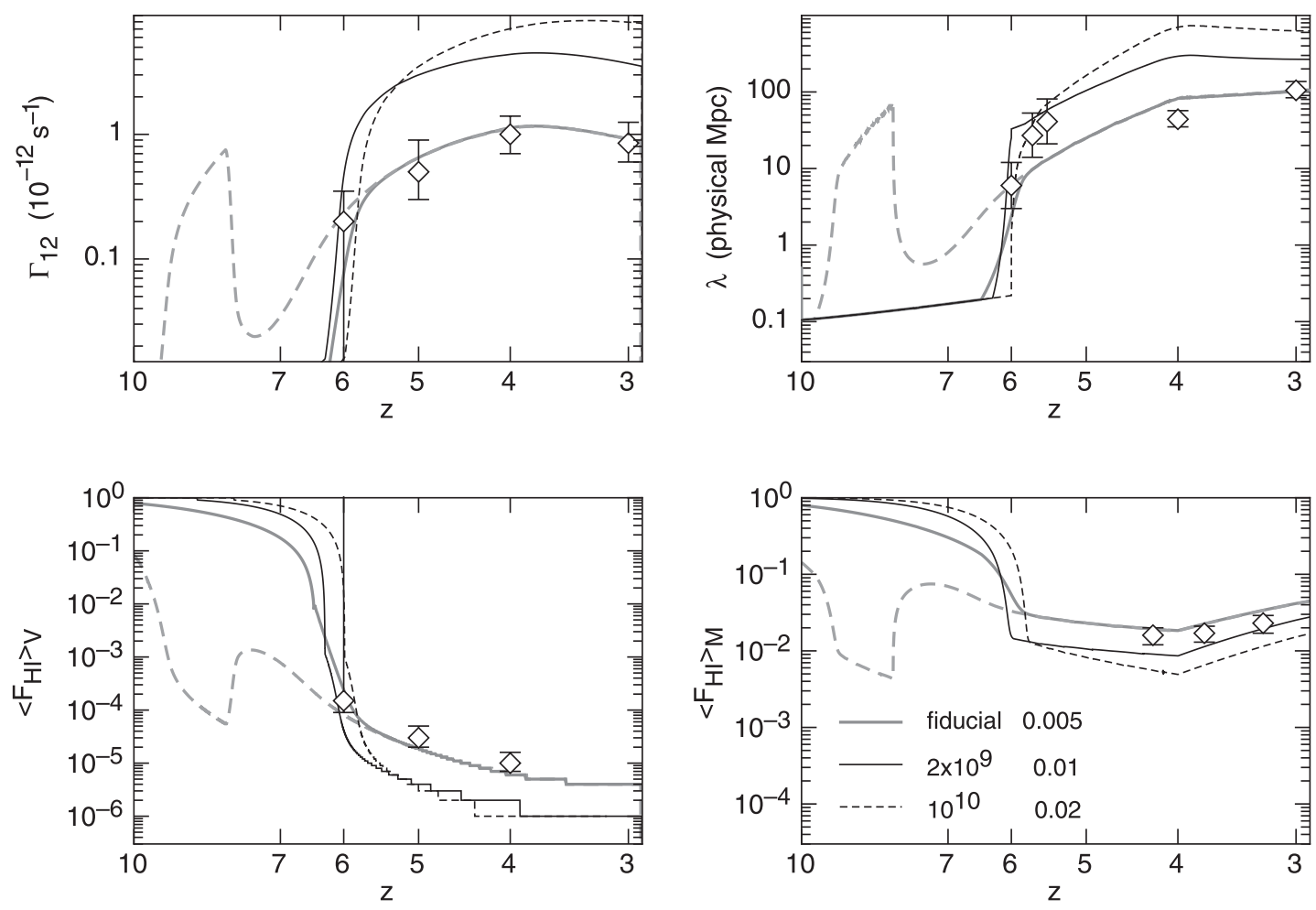

Figure 9 Models for the reionization of the IGM and the subsequent post-overlap evolution of the ionizing radiation field. In each panel the case shown corresponds to a value for the critical over-density prior to the overlap epoch of $\Delta_{\mathrm{c}}=5$. Upper Left Panel: The ionization rate as a function of redshift. The observational points are from Bolton \& Haehnelt (2007). Lower Left and Right Panels: The volume and mass averaged fractions of neutral gas in the universe. The observational points for the volume averaged neutral fraction are from Bolton \& Haehnelt (2007), while the observed mass-fractions are from the damped Ly $\alpha$ measurements of Prochaska et al. (2005). Upper Right Panel: The mean-free-path for ionizing photons. The data points are based on Storrie-Lombardi et al. (1994). The models are labelled by the assumed values of $M_{\text {esc }}$ and $f_{\mathrm{esc}}^{*}$.

Figure 9 shows our fiducial model for the reionization of the IGM and the subsequent post-overlap evolution of the ionizing radiation field, assuming $f_{\text {esc }}^{*}=0.005$ ( $f_{\text {esc }} \sim 5 \%$ ). In the top left panel of Figure 9 we show the evolution of the ionization rate. The observational points are from the simulations of Bolton \& Haehnelt (2007), which at the redshifts shown are based on observations (Songaila 2004; Fan et al. 2006) and simulated data (Schaye et al. 2003) of the Ly $\alpha$ opacity of the IGM. The assumed value of $f_{\mathrm{esc}}^{*}=0.005$ provides the best fit to the data, and is consistent with modelling of the $\operatorname{LF}\left(f^{\star} \sim 0.1\right)$ as well as extrapolation of the escape fraction to minimum masses below $10^{9} \mathrm{M}_{\odot}\left(f_{\text {esc }} \sim 5 \%\right)$.

In the lower-left and lower-right panels we plot the corresponding volume and mass (upper curves) averaged fractions of neutral gas in the universe. The observational points for the volume averaged neutral fraction are from Bolton \& Haehnelt (2007), while the observed mass-fractions are from the damped Ly $\alpha$ measurements of Prochaska, Herbert-Fort \& Wolfe (2005), and therefore represent lower limits on the total HI content of the IGM. Both curves for the mass fraction and the volume fraction show excellent agreement with these observed quantities. This is despite their differing by 3 orders of magnitude and indicates the applicability of the model over a wide density range. In the upper-right panel we plot the evolution of the ionizing photon mean-free-path. The data points at $z>5$ are based on Storrie-Lombardi et al. (1994). At $z>5$ the values of the MFP adopted in our simulations are shown for comparison. Again the model is in good agreement with the available observations and the estimated MFPs. The observed values for the MFP obtained by StorrieLombardi et al. (1994) are found from the number density of Lyman-limit systems (which represents an upper limit, see discussion in Section 3.2) and is independent of the quantities of ionization rate and volume-averaged neutral fraction derived from Ly $\alpha$ Forest absorption, as well as being independent of the Hi mass-density measurements. Our simple model therefore simultaneously reproduces the evolution of three independent measured quantities.

The agreement of this model with observations is an important consistency check of our parameter estimates in previous sections. Taken together, our results for the escape fraction and star-formation efficiency based on our numerical simulations combined with the model reionization histories suggest that the observed transmission and LF are compatible with reionization at $z \sim 6$.

\subsection{Comparison of the Escape Fraction in Numerical and Semi-analytic Models}

Our numerical simulations are only able to resolve galaxies down to a halo mass of $M=2 \times 10^{9} \mathrm{M}_{\odot}$, while in our fiducial reionization model the bulk of ionizing photons prior to reionization come from lower mass galaxies 
$\left(M \gtrsim 10^{8} \mathrm{M}_{\odot}\right)$. We therefore also use our semi-analytic history model to investigate the implications of excluding these lower mass galaxies, as well as galaxies with halo mass below $10^{10} \mathrm{M}_{\odot}$ (see Section 4). We adopt values for the escape fraction of $f_{\text {esc }}^{*}=0.01$ and $f_{\text {esc }}^{*}=0.02$ respectively, which are consistent with our numerical results in the previous sections. The results of these models are plotted in Figure 9 (thin and dashed lines). These histories are consistent with reionization at $z \sim 6$, which directly constrains the result that suggests that the observed transmission and LF are compatible with reionization at $z \sim 6$. However, these models exceed the observed ionization rate at $z \lesssim 6$, owing to the rapid increase in the collapsed fraction.

In addition to the properties described in Figure 9, reionization histories are also constrained by the optical depth to Thomson scattering of CMB photons. The fiducial model in Figure 9 has an optical depth of $\tau_{\mathrm{es}}=0.063$, while models with $M_{\mathrm{esc}}=10^{9} \mathrm{M}_{\odot}$ and $M_{\mathrm{esc}}=10^{10} \mathrm{M}_{\odot}$ have $\tau_{\mathrm{es}}=0.045, \tau_{\mathrm{es}}=0.041$. These values are smaller than the value determined from the WMAP satellite, $\tau_{\mathrm{es}}=0.084 \pm 0.016$ (Komatsu et al. 2009).

\subsection{Reionization Histories with Massive Stars}

Before concluding, we consider the implications of a population of massive stars at high redshift. Models which include massive Population-III stars partially reionize the IGM at high redshift and result in larger values of $\tau_{\mathrm{es}}$. For example, in the work of Choudhury \& Ferrara (2005) and Wyithe \& Cen (2007), models in which the reionization history shows an extended plateau of mostly ionized IGM in the range $6 \lesssim z \lesssim 10$ are able to produce values of $\tau_{\mathrm{es}}$ within the preferred range plus completion of reionization at $z \sim 6$. This early reionization does not however make our predicted values of the escape fraction inconsistent with reionization at $z \sim 6$.

The heavy dashed line in Figure 9 represents a model for the reionization history which includes Population-III stars at high redshift. Here we assume that the value of $f_{\text {esc }}^{*}$ is not sensitive to redshift, but that 10 times the number of ionizing photons per baryon are emitted prior to a transition redshift $z_{\text {tran }}$. Note that this modelling is intended to be illustrative only and does not include more advanced treatments of the transition redshift, such as those studied by Schneider et al. (2006), Scannapieco, Schneider \& Ferrara (2003) and Wyithe \& Cen (2007). The example shown illustrates the effect of a high redshift population of massive stars and corresponds to an assumed transition redshift of $z_{\text {tran }}=8$. Finally, the addition of PopulationIII stars increases the value of $\tau_{\mathrm{es}}$. For the inclusion of Population-III stars in our fiducial model, where $z_{\operatorname{tran}}=8$, we find $\tau_{\mathrm{es}}=0.095$, which is consistent with the results from the WMAP satellite ( $\left.\tau_{\mathrm{es}}=0.084 \pm 0.016\right)$.

\section{Conclusions}

In this paper we have employed an $N$-body simulation to describe the density and peculiar velocity fields in a $(65.6 \mathrm{Mpc} / h)^{3}$ volume of the IGM at $z=5.5,5.7,6.0$.
The simulation also identified the locations and masses of virialized halos, with the minimum halo mass accurately resolved by the simulation being $2 \times 10^{9} M_{\odot}$. We associate the identified halos with galaxies and assign to them a UV luminosity using models for the SFR and the SED. By fitting to the observed UV galaxy LF (Bouwens et al. 2007) we constrained the best fit free parameters to be $f^{\star}=0.11$ and $t_{\mathrm{DC}}=0.16$, where $f^{\star}$ is the fraction of baryons which participate in star formation and $t_{\mathrm{DC}}$ is the duty-cycle of star-bursting galaxies. We then constructed an ionizing background which reflects the spatial distribution of UV luminous galaxies. Our model made the assumption of a universal MFP at each redshift.

Given the density, velocity and ionizing radiation fields we extracted an ensemble of Ly $\alpha$ absorption spectra along 3072 sight-lines. From this sample we computed the mean effective transmission as a function of the fraction of ionizing photons produced that escape their host galaxy $\left(f_{\text {esc }}\right)$. In our simulations $f_{\text {esc }}$ effectively adjusts the intensity of the ionizing background. The value of $f_{\text {esc }}$ was determined so that the mean effective transmission predicted by the model matched the observed value. Assuming halos above the mass resolution of our simulation to host galaxies, we find values for the escape fraction at $z \sim 5.5-6$ of $f_{\text {esc }} \sim 10-25 \%$ assuming values for the MFP which are suggested by (Fan et al. 2006). In models where we assume the formation of galaxies to be suppressed in halos with masses below $10^{10} \mathrm{M}_{\odot}$ (possibly due to radiation feedback following reionization), we find the escape fraction to be in the range $f_{\text {esc }} \sim 20-40 \%$. Our results for the escape fraction are consistent with those found in Bolton \& Haehnelt (2007) who constrain the escape fraction to be $f_{\text {esc }} \sim 20-30 \%$, using a similar approach to the one adopted in this paper. This is true both for scenarios in which the minimum halo mass is set by the simulation resolution $\left(2 \times 10^{9} \mathrm{M}_{\odot}\right)$ and for the larger value of $10^{10} \mathrm{M}_{\odot}$.

We also considered the scenario suggested by Gnedin et al. (2008) in which the escape fraction of ionizing photons is negligible in halos with masses below $\sim 5 \times 10^{10} \mathrm{M}_{\odot}$. In this case we find the escape fraction is required to be $f_{\text {esc }} \sim 46-86 \%$ in order to reproduce the observed optical depth of the IGM. This is inconsistent with theoretical modelling of the escape fraction by Gnedin et al. (2008) who suggest $f_{\text {esc }} \sim 1-3 \%$. Using the numerical results to calibrate an analytic relation between the escape fraction and minimum galaxy halo mass we extrapolate our results to a mass $\left(M \sim 10^{8} \mathrm{M}_{\odot}\right)$ corresponding to the hydrogen cooling threshold. In this case we find $f_{\text {esc }} \sim 5-10 \%$, consistent with observed estimates at lower redshift.

Since the SFR is estimated at $1350 \AA$, while $f_{\text {esc }}$ is estimated at $912 \AA$, the escape fraction is degenerate with the adopted galaxy SED. The value of the Lyman-break factor $(L B)$, which is the intrinsic flux decrement measured across the Lyman break, is particularly sensitive to the age of the stellar population. Assuming that the normalization of the adopted SED at wavelengths above the Lyman limit, and the slope of the SED at wavelengths below the Lyman 
limit are both correct, we can express this degeneracy with respect to $L B$ as $f_{\mathrm{esc}}=f_{\mathrm{esc}}^{\mathrm{LB}=3}(L B / 3)$. In this expression $f_{\text {esc }}^{\mathrm{LB}=3}$ is the escape fraction which we have constrained in this paper, and $f_{\text {esc }}$ the value obtained for an alternate $L B$.

We use a semi-analytic model that describes the evolution of the ionized state of the post-reionization IGM to study whether the escape fraction implied by our numerical modelling is consistent with reionization at $z \gtrsim 6$. We find that the measured ionization rates imply a history that is consistent with reionization at $z \sim 6$ for a range of these models, including those in which the minimum halo mass of galaxies contributing to the ionizing emissivity is $M \sim 10^{8}, 10^{9}$ and $10^{10} \mathrm{M}_{\odot}$ respectively. However, we find that models with minimum masses above $10^{9} \mathrm{M}_{\odot}$ over-estimate the ionization rate in the post-overlap IGM. These models imply that the Universe was reionized by low mass galaxies with an escape fraction of $\sim 5 \%$.

In summary, the transmission of Ly $\alpha$ photons through the IGM at $z \sim 5.5-6$ implies an ionization rate that requires the escape fraction of ionizing photons to be in excess of $5 \%$. Our results for the escape fraction and starformation efficiency based on our numerical simulations combined with the model reionization histories suggest that the observed transmission and LF are compatible with reionization at $z \sim 6$.

\section{Acknowledgments}

We thank Adam Lidz for providing the $N$-body simulation and technical assistance, and also the Institute for Theory and Computation at CfA (Harvard-Smithsonian) for use of their computational facilities during this project. This work was supported in part by the Australian Research Council. J.A.S. acknowledges the support of an Australian Postgraduate Award.

\section{References}

Alvarez, M. A., Bromm, V. \& Shapiro, P. R., 2006, ApJ, 639, 621 Barkana, R. \& Loeb, A., 2001, ApJS, 349, 125

Bergvall, N., Zackrisson, E., Andersson, B.-G., Arnberg, D., Masegosa, J. \& Östlin, G., 2006, A\&A, 448, 513

Bland-Hawthorn, J. \& Maloney, P. R., 1999, ApJ, 510, L33

Bolton, J. S. \& Haehnelt, M. G., 2007, MNRAS, 382, 325

Bolton, J. S., Haehnelt, M. G., Viel, M. \& Springel, V., 2005, MNRAS, 357, 1178

Bond, J. R., Cole, S., Efstathiou, G. \& Kaiser, N., 1991, ApJ, 379, 440

Bouwens, R. J., Illingworth, G. D., Franx, M. \& Ford, H., 2007, ApJ, 670, 928

Chen, H.-W., Prochaska, J. X. \& Gnedin, N. Y., 2007, ApJ, 667, L125

Choudhury, T. R. \& Ferrara, A., 2005, MNRAS, 361, 577

Ciardi, B., Bianchi, S. \& Ferrara, A., 2002, MNRAS, 331, 463

Clarke, C. \& Oey, M. S., 2002, MNRAS, 337, 1299

Croft, R. A. C., 2004, ApJ, 610, 642

Deharveng, J.-M., Buat, V., Le Brun, V., Milliard, B., Kunth, D., Shull, J. M. \& Gry, C., 2001, A\&A, 375, 805

Dijkstra, M., Haiman, Z., Rees, M. J. \& Weinberg, D. H., 2004, ApJ, 601, 666

Dove, J. B. \& Shull, J. M., 1994, ApJ, 430, 222

Dove, J. B., Shull, J. M. \& Ferrara, A., 2000, ApJ, 531, 846

Efstathiou, G., 1992, MNRAS, 256, 43

Fan, X., Narayanan, V. K., Strauss, M. A., White, R. L., Becker, R. H., Pentericci, L. \& Rix, H. W., 2002, AJ, 123, 1247

Fan, X. et al., 2006, AJ, 132, 117
Faucher-Giguere, C., Lidz, A., Hernquist, L., Zaldarriaga, M. \& 2008, ApJ, 688, 85

Fernández-Soto, A., Lanzetta, K. M. \& Chen, H.-W., 2003, MNRAS, 342,1215

Fujita, A., Martin, C. L., Mac Low, M.-M. \& Abel, T., 2003, ApJ, 599,50

Furlanetto, S. R. \& Oh, S. P., 2005, MNRAS, 363, 1031

Giallongo, E., Cristiani, S., D’Odorico, S. \& Fontana, A., 2002, ApJ, 568, L9

Gnedin, N. Y., 2008, ApJL, 673, L1

Gnedin, N. Y. \& Hamilton, A. J. S., 2002, MNRAS, 334, 107

Gnedin, N. Y. \& Hui, L., 1998, MNRAS, 296, 44

Gnedin, N. Y., Kravtsov, A. V. \& Chen, H.-W., 2008, ApJ, 672, 765

Grimes, J. P. et al., 2007, ApJ, 668, 891

Heckman, T. M., Sembach, K. R., Meurer, G. R., Leitherer, C., Calzetti, D. \& Martin, C. L., 2001, ApJ, 558, 56

Hui, L., Gnedin, N. Y. \& Zhang, Y., 1997, ApJ, 486, 599

Hurwitz, M., Jelinsky, P. \& Dixon, W. V. D., 1997, ApJ, 481, L31

Inoue, A. K., Iwata, I. \& Deharveng, J.-M., 2006, MNRAS, 371, L1

Inoue, A. K., Iwata, I., Deharveng, J.-M., Buat, V. \& Burgarella, D., 2005, A\&A, 435, 471

Kitayama, T., Yoshida, N., Susa, H. \& Umemura, M., 2004, ApJ, 613, 631

Komatsu, E. et al., 2009, ApJS, 180, 330

Lai, K., Lidz, A., Hernquist, L. \& Zaldarriaga, M., 2006, ApJ, 644, 61

Leitherer, C. et al., 1999, ApJS, 123, 3

Lidz, A., Oh, S. P. \& Furlanetto, S. R., 2006, ApJ, 639, L47

Lidz, A., Zahn, O., McQuinn, M., Zaldarriaga, M., Dutta \& S. Hernquist, L., 2007, ApJ, 659, 865

Loeb, A., Barkana, R. \& Hernquist, L., 2005, ApJ, 620, 553

Madau, P., Haardt, F. \& Rees, M. J., 1999, ApJ, 514, 648

Malkan, M., Webb, W. \& Konopacky, Q., 2003, ApJ, 598, 878

McDonald, P., Seljak, U., Cen, R., Bode, P. \& Ostriker, J. P., 2005, MNRAS, 360, 1471

McQuinn, M., Lidz, A., Zahn, O., Dutta, S., Hernquist, L. \& Zaldarriaga, M., 2007, MNRAS, 377, 1043

Meiksin, A. \& White, M., 2004, MNRAS, 350, 1107

Miralda-Escudé, J., 2003, ApJ, 597, 66

Miralda-Escudé, J., Haehnelt, M. \& Rees, M. J., 2000, ApJ, 530, 1

Press, W. H. \& Schechter, P., 1974, ApJ, 187, 425

Prochaska, J. X., Herbert-Fort, S. \& Wolfe, A. M., 2005, ApJ, 635, 123

Razoumov, A. O. \& Sommer-Larsen, J., 2006, ApJ, 651, L89

Scalo, J., 1998, ASPC, 142, 201

Scannapieco, E., Schneider, R. \& Ferrara, A., 2003, ApJ, 589, 35

Schaye, J., 2006, ApJ, 643, 59

Schaye, J., Aguirre, A., Kim, T.-S., Theuns, T., Rauch, M. \& Sargent, W. L. W., 2003, ApJ, 596, 768

Schneider, R., Salvaterra, R., Ferrara, A. \& Ciardi, B., 2006, MNRAS, 369, 825

Shapley, A. E., Steidel, C. C., Pettini, M., Adelberger, K. L. \& Erb, D. K., 2006, ApJ, 651, 688

Sheth, R. K. Tormen, G., 1999, MNRAS, 308, 119

Siana, B. et al., 2007, ApJ, 668, 62

Songaila, A., 2004, AJ, 127, 2598

Srbinovsky, J. A. \& Wyithe, J. S. B., 2007, MNRAS, 374, 627

Steidel, C. C., Pettini, M. \& Adelberger, K. L., 2001, ApJ, 546, 665

Storrie-Lombardi, L. J., McMahon, R. G., Irwin, M. J. \& Hazard, C., 1994, ApJ, 427, L13

Thoul, A. A. \& Weinberg, D. H., 1996, ApJ, 465, 608

Whalen, D., Abel, T. \& Norman, M. L., 2004, ApJ, 610, 14

Wood, K. \& Loeb, A., 2000, ApJ, 545, 86

Wyithe, J. S. B. \& Cen, R., 2007, ApJ, 659, 890

Wyithe, J. S. B. \& Loeb, A., 2003, ApJ, 586, 693

Wyithe, S., Bolton, J. \& Haehnelt, M., 2007, ArXiv e-prints, 708

Zahn, O., Lidz, A., McQuinn, M., Dutta, S., Hernquist, L., Zaldarriaga, M. \& Furlanetto, S. R., 2007, ApJ, 654, 12 